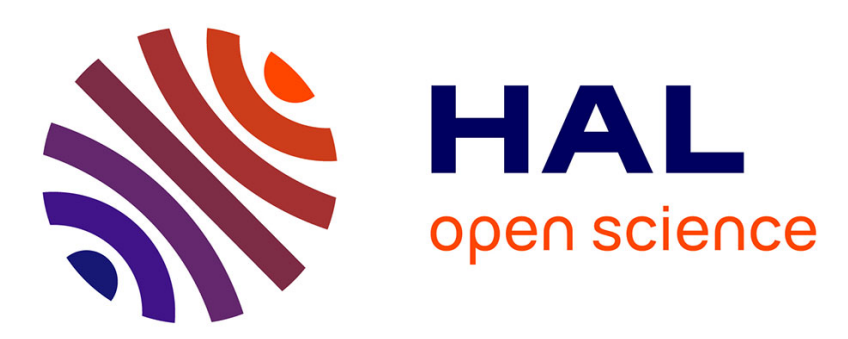

\title{
Méthodologie pour le dimensionnement à l'impact d'un empilement linéaire élastique et viscoélastique
}

\author{
B. Vidal, J.-L. Lataillade, Francis Collombet, C. Bacon
}

\section{To cite this version:}

B. Vidal, J.-L. Lataillade, Francis Collombet, C. Bacon. Méthodologie pour le dimensionnement à l'impact d'un empilement linéaire élastique et viscoélastique. Journal de Physique IV Proceedings, 1997, 07 (C3), pp.C3-779-C3-784. 10.1051/jp4:19973132 • jpa-00255419

\section{HAL Id: jpa-00255419 https://hal.science/jpa-00255419}

Submitted on 1 Jan 1997

HAL is a multi-disciplinary open access archive for the deposit and dissemination of scientific research documents, whether they are published or not. The documents may come from teaching and research institutions in France or abroad, or from public or private research centers.
L'archive ouverte pluridisciplinaire HAL, est destinée au dépôt et à la diffusion de documents scientifiques de niveau recherche, publiés ou non, émanant des établissements d'enseignement et de recherche français ou étrangers, des laboratoires publics ou privés. 


\title{
Méthodologie pour le dimensionnement à l'impact d'un empilement linéaire élastique et viscoélastique
}

\author{
B. Vidal, J.-L. Lataillade, F. Collombet et C. Bacon* \\ LAMEF-École Nationale Supérieure d'Arts et Métiers, Esplanade des Arts et Métiers, \\ 33405 Talence cedex, France \\ * LMP-Université Bordeaux 1, 351 Cours de la Libération, 33405 Talence, France
}

\begin{abstract}
Résumé. Une méthode pour dimensionner un assemblage plan collé face à un impact de type basse vitesse est présentée. Une modélisation analytique de l'impact permet un prédimensionnement rapide de l'assemblage. Les dimensions optimales sont finalement déterminées avec un modèle éléments finis qui décrit le contact au point d'impact. Le comportement de la colle est identifié avec un viscoélasticimètre puis implémenté dans le code numérique. Ces modèles se révélant proches de l'expérience, cette méthode devrait permettre de gagner du temps en diminuant considérablement les essais.
\end{abstract}

\begin{abstract}
In order to size a glued plane assembly under low velocity impact, a method has been developed. An analytical model gives us a quick design of the optimised dimensions. A finite element model describes the impact mode contact. A viscoelasticimeter gives glue behaviour which is, then, implemented in the numerical code. These models are very close to experiment, so this method will save time by reducing the number of tests.
\end{abstract}

\section{INTRODUCTION}

Les assemblages plans collés peuvent être endommagés sous forme de délaminage localisés par des impacts de type basses vitesses. Pour une telle structure, lorsqu'il s'agit de définir ses dimensions transversales, son empilement ou ses conditions de fixation, l'approche expérimentale devient vite longue et coûteuse. Si elle reste indispensable, il faut se donner les moyens d'en diminuer le coût.

La modélisation numérique par éléments finis est un outil puissant pour résoudre ce type de problème. Cependant, elle manque souvent de souplesse pour explorer de vastes domaines dimensionnels. Cette modélisation n'est efficace en fait que lorsque le domaine de recherche est déjà assez restreint. Il est donc nécessaire d'utiliser, en amont, des outils simples qui permettent de localiser rapidement les dimensions optimales de l'assemblage.

Nous développons ici une méthode de dimensionnement qui vise à garder l'intégrité de la structure face à ce type d'agression.

Un modèle analytique nous permet de cerner rapidement la solution du problème. Ces structures contiennent des couches de polymères dont le comportement thermoviscoélastique est à prendre en compte. En utilisant un viscoélasticimètre, nous identifions ces comportements viscoélastique. Ces comportements sont implémentés dans un modèle d'impact de type éléments finis. La recherche des dimensions optimales de la structure est effectuée, avec ce modèle éléments finis, sur un domaine limité au voisinage de la solution approchée avec le modèle analytique.

\section{DISPOSITIF EXPERIMENTAL}

Une bille d'acier est projetée à faible vitesse $(\mathrm{Vi}<5 \mathrm{~m} / \mathrm{s})$ sur une plaque circulaire de $30 \mathrm{~cm}$ de diamètre et de $4 \mathrm{~mm}$ d'épaisseur (Fig. 1).Cela autorise une interaction matériau-structure. L'instrumentation du dispositif permet de mesurer la déformation et le déplacement au point opposé à l'impact ainsi que la force exercée par le projectile. La fermeture d'un circuit électrique lors du contact entre la bille et le disque permet de repérer précisément les instants de contact. C'est ainsi que l'on observe de multiples pertes et reprises de contact durant le même impact (Fig. 2) 


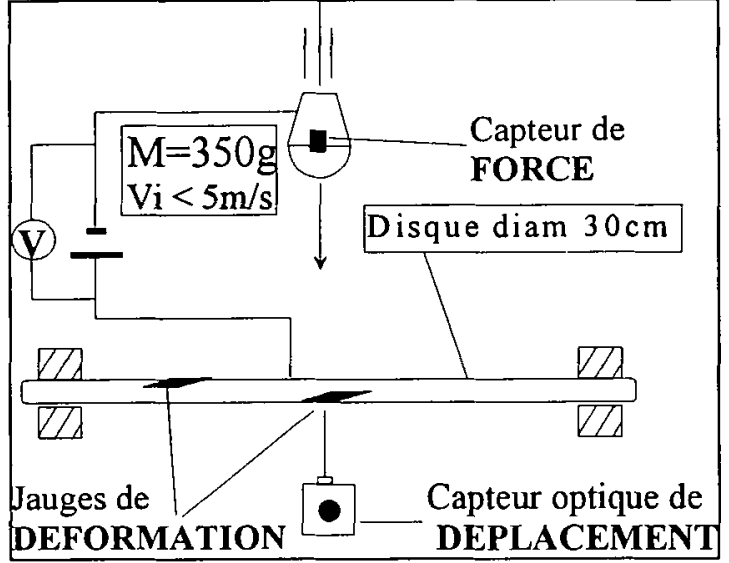

Figure 1: Dispositif expérimental

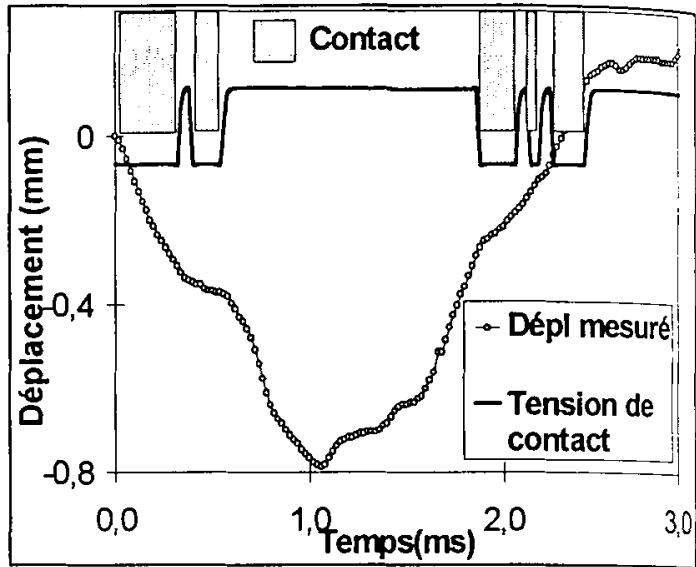

Figure 2: Repérage des instants de contact

\section{MODELISATION ANALYTIQUE}

Pour tenir compte de ce résultat expérimental, nous décrivons analytiquement le choc comme une succession de contacts et pertes de contact. Nous utilisons l'analyse modale de deux systèmes [1] [2]. Durant le contact, le mouvement libre du système lié plaque-projectile est décrit par un premier modele. Une fois le contact rompu, c'est un second modèle qui permet de représenter la vibration libre de la plaque. Cette modélisation, permet d'atteindre les déplacements du projectile et de la plaque ainsi que la force de contact du projectile sur la plaque. En raison de la triaxialité du champ des contraintes au point d'impact, le calcul biaxial de ce champ ne trouve un sens qu'en dehors du voisinage de ce point.

En confrontant ce modèle analytique à l'expérience et à un modèle éléments finis, nous montrons sa validité (Fig.3 et Fig. 4), preuve que les phénomènes engendrés sous la bille n'ont pas de répercussion sur la réponse globale.

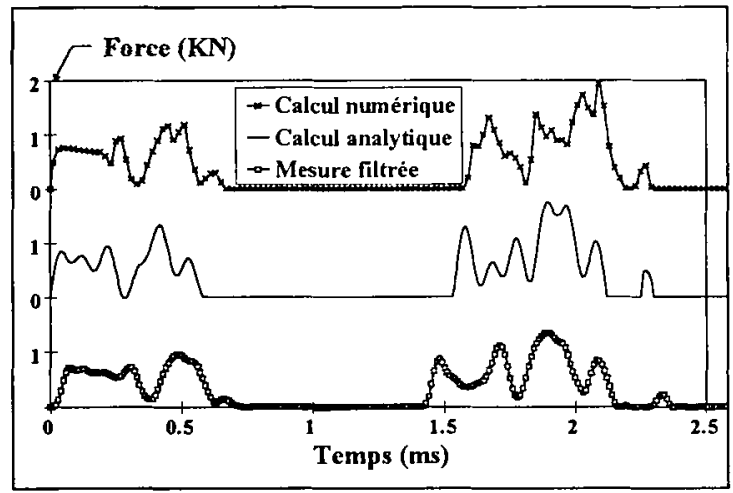

Figure 3: Force d'impact au centre du disque

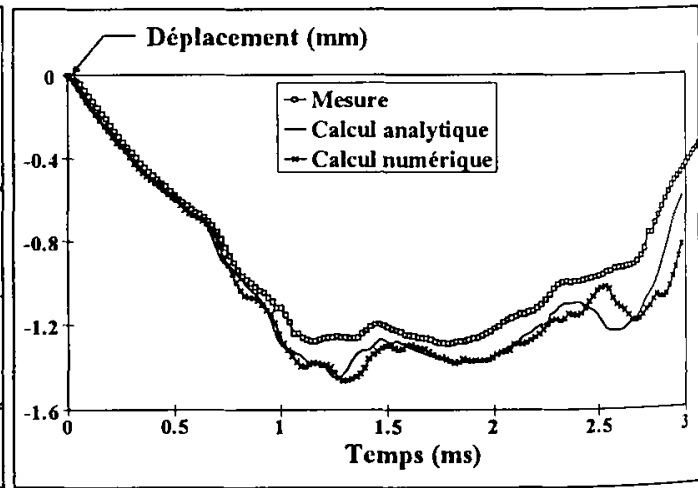

Figure 4: Déplacement au centre du disque

La bonne corrélation calcul analytique-mesure permet d'utiliser ce modèle pour évaluer l'influence des différents paramètres que sont la vitesse d'impact, la masse du projectile, les conditions de fixation et les dimensions de la structure. En utilisant un critère fondé sur les contraintes et la force de contact maximales admissibles précédant la dégradation de la structure, les dimensions optimales seront rapidement localisées 


\section{RHEOLOGIE DES POLYMERES}

Les polymères présents dans l'assemblage ont un comportement viscoélastique qui les rend sensibles à la température et à la vitesse de déformation. Le domaine de sollicitation de ces polymères est, dans le cas d'un impact, celui des grandes vitesses de déformation $\left(\cong 10 \mathrm{~m}(\mathrm{~ms})^{-1}\right)$.

La caractérisation du comportement viscoélastique étant complexe, les données concernant ces polymères sont souvent rares ou incomplètes.

Ces informations sont pourtant capitales pour l'étude des impacts sur ces assemblages. Traduites sous forme de modèles rhéologiques, ces mesures peuvent être implémentées dans des codes numériques pour simuler les impacts et prédire ainsi le comportement de la structure en fonction de la température et des vitesses d'impact.

\subsection{Mesure du comportement viscoélastique}

La réponse viscoélastique des matériaux présents dans l'empilement est mesurée en cisaillement pour différentes fréquences et températures à l'aide d'un viscoélasticimètre. L'application du principe d'équivalence fréquence-température permet d'identifier les modules de cisaillement réels et imaginaires, $G^{\prime}$ et G', sur une plage de fréquences allant jusqu'à $10 \mathrm{MHz}$. Cela permet de couvrir largement le spectre de fréquences du choc étudié. (Nous faisons ici l'hypothèse de comportements linéaires)

\subsection{Modèle de MAXWELL généralisé}

De nombreux codes numériques traitant des phénomènes transitoires dans les matériaux viscoélastiques, modélisent le comportement en cisaillement de ces structures sous la forme du modèle de MAXWELL généralisé. La fonction relaxation $G(t)$ est décrite par $N+1$ coefficients $G_{i}$ et $N$ temps de relaxation $\tau_{i}(1)$.

$$
G(t)=G_{0}+\sum_{i=1}^{N} G_{i} e^{-\left(\frac{t}{\tau_{i}}\right)}
$$

Lors d'une déformation, $\gamma(\mathrm{t})$, sinusoïdale de pulsation $\omega$, la contrainte de cisaillement, $\tau(\mathrm{t})$, devient [3]:

$$
\begin{aligned}
& \gamma(t)=\gamma o \sin (\omega t) \\
& \tau(t)=\gamma o\left[G^{\prime}(\omega) \sin (\omega t)+G^{\prime \prime}(\omega) \cos (\omega t)\right]
\end{aligned}
$$

avec, dans le cas d'une modélisation de type MAXWELL Généralisé :

$$
\begin{aligned}
& G^{\prime}(\omega)=G_{0}+\sum_{i=1}^{N} G_{i} \frac{\left(\omega \tau_{i}\right)^{2}}{1+\left(\omega \tau_{i}\right)^{2}} \\
& G^{\prime \prime}(\omega)=\sum_{i=1}^{N} G_{i} \frac{\omega \tau_{i}}{1+\left(\omega \tau_{i}\right)^{2}}
\end{aligned}
$$

Les paramètres $\tau_{i}$ et $G_{i}$ sont évalués par une minimisation non linéaire de l'écart entre les résultats des équations (3) et les mesures [4] [5].

Les modules de cisaillement réel et imaginaire, $G^{\prime}$ et $G^{\prime \prime}$, provenant de ce modèle se montrent proches de ceux déduits de l'expérience par le principe d'équivalence fréquence-température (Fig. 5). Le calcul de temps de relaxation de l'ordre de $0.1 \mu$ s permet de couvrir le spectre de fréquences exploré expérimentalement. 


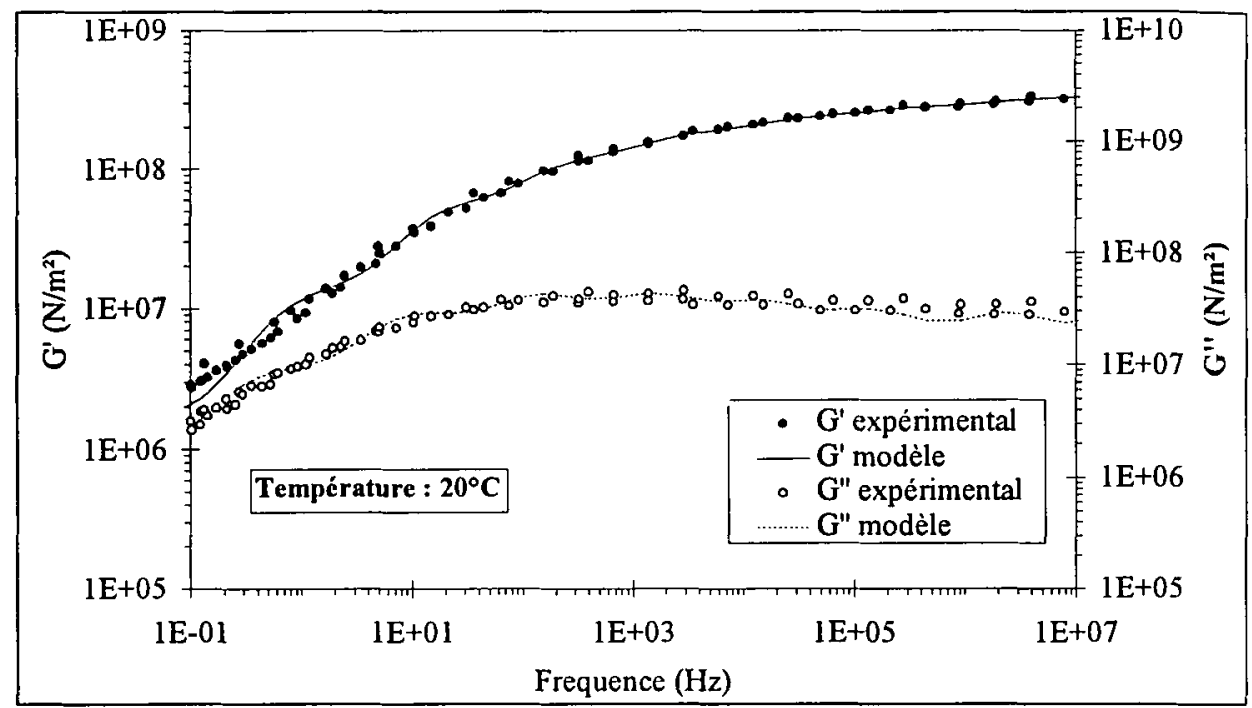

Figure 5: Comparaison Mesure-Modèle de MAXWELL

\section{MODELISATION NUMERIQUE}

La réponse viscoélastique des matériaux testés est donc introduite, sous la forme du modèle de

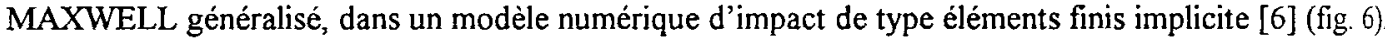

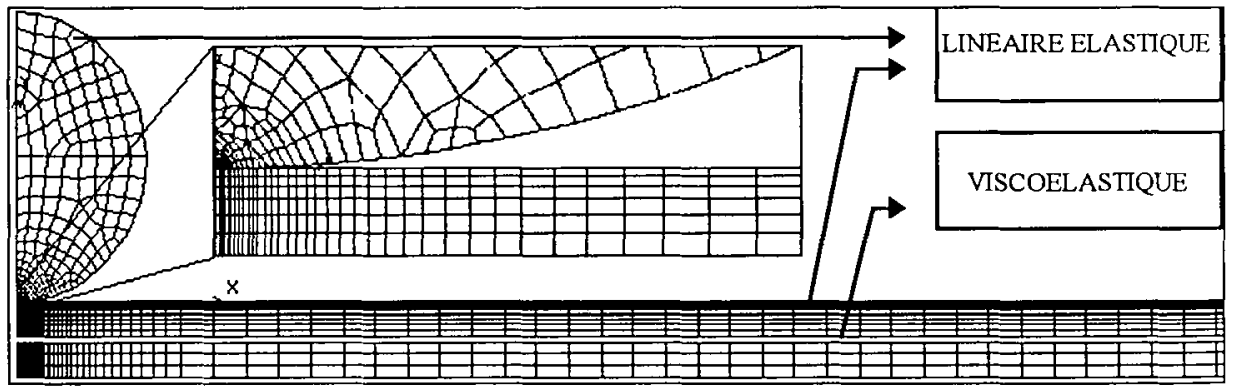

Figure 6: Modèle numérique axisymétrique d'impact

\subsection{Validation analytique du contact}

L'endroit du contact entre la bille et l'assemblage est une zone soumise à de forts gradients de contraintes qui peuvent conduire à l'endommagement de la structure. Il est donc important de valider les efforts calculés en ce lieu. Cependant, les mesures de déformations y sont très complexes à réaliser.

La démarche suivie revient à considérer que ce champ de contraintes s'apparente à celui décrit par Hertz en statique [7](contact de la sphère sur un plan). Sachant que le traitement numérique du contact est le même en dynamique et en statique, la loi de Hertz est modélisée numériquement puis confrontée au calcul analytique (fig. 7). La bonne corrélation calcul-théorie valide, en statique, d'une part la gestion numérique du contact et d'autre part le choix du maillage dans la région du contact. L'utilisation, pourle modèle d'impact, de ce même maillage dans la zone de contact permet de contrôler la validité du calcul. 


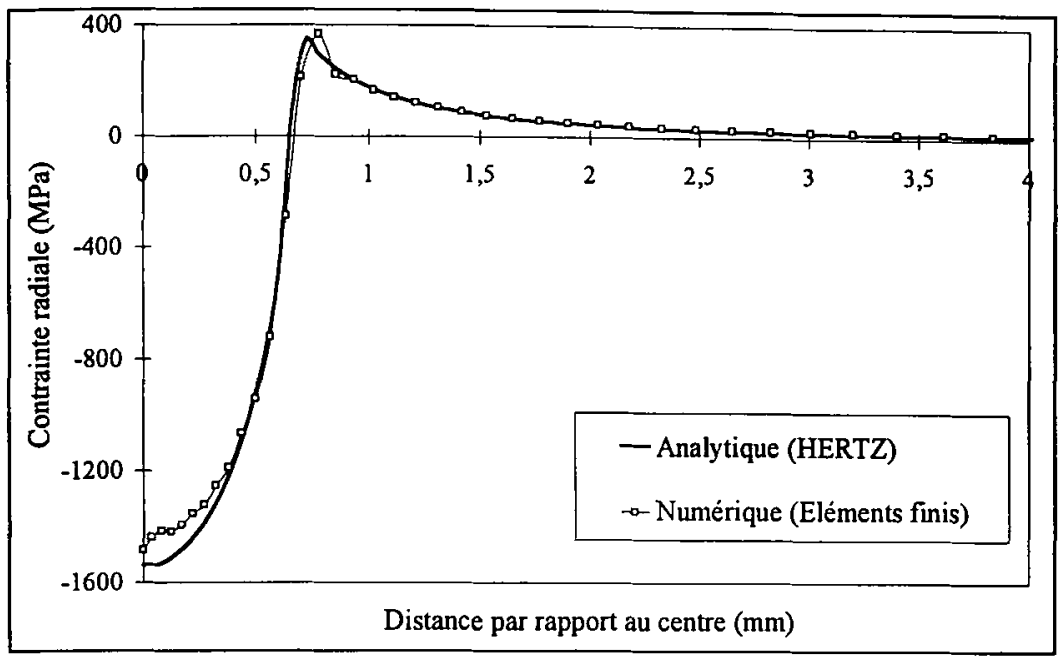

Figure 7: Indentation statique d'une sphère sur un plan : comparaison Théorie de HERTZ-Modèle numérique

\subsection{Validation expérimentale du modèle}

La confrontation avec l'expérience (fig. 8 et 9) rend compte de la validité de ce modèle dans le cadre des impacts survenant à basse vitesse sur des assemblages constitués de matériaux à comportement linéaire élastique et viscoélastique. Le calcul des déplacements et des déformations se révèle proche des mesures. Pour dimensionner l'assemblage, le résultat du calcul peut être exploité sur toute la structure et même dans la zone de contact. L'identification expérimentale de critères d'endommagement des matériaux permettra, une fois implémentée dans ce modèle, de prédire les conditions de dégradation de l'assemblage et donc d'en optimiser la géométrie.

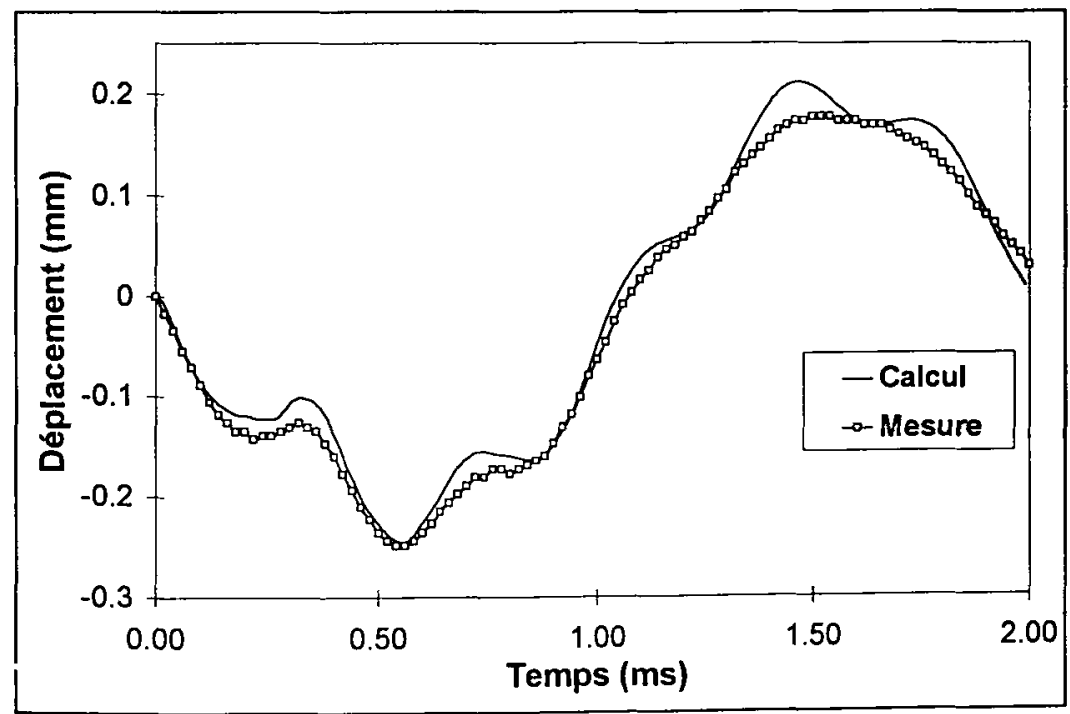

Figure 8: Déplacement du centre du disque 


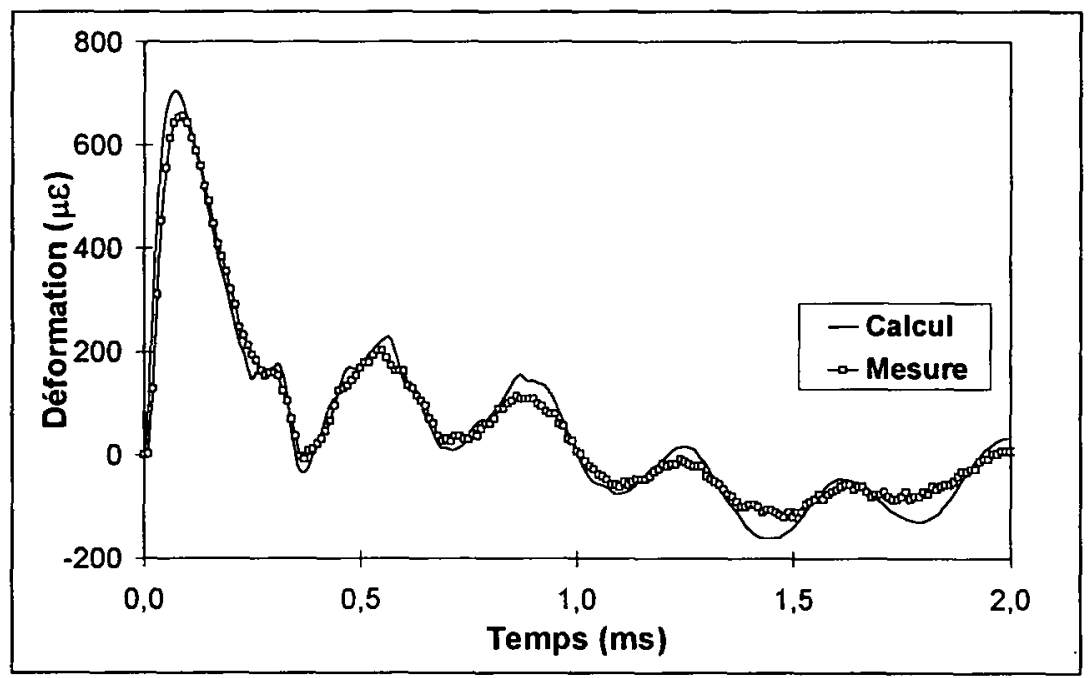

Figure 9: Déformation radiale au centre du disque

\section{CONCLUSION}

Nous venons de présenter les outils que nous utiliserons pour dimensionner les assemblages plans colles. Les modèles analytique et numérique ainsi que la modélisation du comportement viscoélastique des couches de polymères se révèlent proches des expériences. La modélisation numérique du contact est validée analytiquement en statique par la solution de Hertz. Ces outils permettent de traiter les impacts sur des empilements en matériaux linéaires élastiques et viscoélastiques.

L'un des buts du dimensionnement aux chocs est de garder l'intégrité de la structure. Pour réaliser cet objectif, la suite de nos travaux s'oriente vers l'identification expérimentale des critères d'endommagement: des matériaux constitutifs de l'assemblage. La prise en compte de ces critères dans nos modèles permettra d'approcher rapidernent une géométrie optimale et le nombre d'essais en sera considérablement diminué.

\section{Références}

[1] Lin R. M. and Lim M. K., Computers and Structures, 57 (1995) 721-729.

[2] Wu E., Tsai T. D. and Yen C. S., Experimental Mechanics, (March 1995) 11-18.

[3] Orbey N. and Dealy M., Journal of Rheology, 35(1991) 1035-1049.

[4] C. L. Lawson and R. J. Hanson, Solving Least Squares Problems (Prentice-Hall, 1974) Chap. 23.

[5] Nelder J. A. and Mead R., Computer Journal, 7, 308-313.

[6] COSMOS/M version 1.75 Finite Element Analysis, The Advanced Modules, (1996).

[7] Timoshenko S. et Goodier J.N., Theory of elasticity $2^{\text {nd }}$ edition (McGraw Hill, New York, 1951). 